\title{
Intuitionistic Fuzzy Soft Matrix Theory
}

\author{
Md.Jalilul Islam Mondal*, Tapan Kumar Roy \\ Department of Mathematics, Bengal Engineering and Science University,Shibpur, Howrah- 711103, West Bengal, India \\ *Corresponding Author: ji.mondal@gmail.com
}

Copyright $(2013$ Horizon Research Publishing All rights reserved.

\begin{abstract}
The purpose of this paper is to put forward the notion of intuitionistic fuzzy soft matrix theory and some basic results. In this paper, we define intuitionistic fuzzy soft matrices and have introduced some new operators with weights, some properties and their proofs and examples which make theoretical studies in intuitionistic fuzzy soft matrix theory more functional. Moreover, we have given one example on weighted arithmetic mean for decision making problem.
\end{abstract}

Keywords Soft Sets, Fuzzy Soft Matrices, Intuitionistic Fuzzy Soft Matrices

\section{Introduction}

Most of our traditional tools for formal modeling, reasoning, and computing are crisp, deterministic, and precise in character. However, in real life, there are many complicated problems in engineering, economics, environment, social sciences medical sciences etc. that involve data which are not all always crisp, precise and deterministic in character because of various uncertainties typical problems. Such uncertainties are being dealing with the help of the theories, like theory of probability, theory of fuzzy sets, theory of intuitionistic fuzzy sets, theory of interval mathematics and theory of rough sets etc. Molodtsov [1] also described the concept of "Soft Set Theory" having parameterization tools for dealing with uncertainties. Researchers on soft set theory have received much attention in recent years. Maji and Roy [3] first introduced soft set into decision making problems. Maji et al.[6] introduced the concept of fuzzy soft sets by combining soft sets and fuzzy sets. Cagman and Enginoglu [4] defined soft matrices which were a matrix representation of the soft sets and constructed a soft max-min decision making method . Borah et al.[8] extended fuzzy soft matrix theory and its application. Deli and Cagmam[9] introduced intuitionistic fuzzy parameterized soft sets. They have also applied to the problems that contain uncertainties based on intuitionistic fuzzy parameterized soft sets. Babitha and John[11] described generalized intuitionistic fuzzy soft sets and solved multi criteria decision making problem in generalized intuitionistic fuzzy soft sets . Rajarajeswari and Dhanalakshmi[10] described intuitionistic fuzzy soft matrix with some traditional operations.

In this paper, we have introduced some operators on intuitionistic fuzzy soft matrix on the basis of weights. We have also discussed their properties . Finally we have given an elementary application on decision making problem on the basis of weighted arithmetic mean.

\section{Definition and Preliminaries}

\subsection{Soft Set [1]}

Let $\mathrm{U}$ be an initial universe, $\mathrm{P}(\mathrm{U})$ be the power set of $\mathrm{U}, \mathrm{E}$ be the set of all parameters and $\mathrm{A} \subseteq \mathrm{E}$. A soft set $\left(f_{A}, \mathrm{E}\right)$ on the universe $U$ is defined by the set of order pairs

$$
\left(f_{A}, \mathrm{E}\right)=\left\{\left(\mathrm{e}, f_{A}(\mathrm{e})\right): \mathrm{e} \in \mathrm{E}, f_{A}(\mathrm{e}) \in \mathrm{P}(\mathrm{U})\right\}
$$

where $f_{A}: \mathrm{E} \rightarrow \mathrm{P}(\mathrm{U})$ such that $f_{A}(\mathrm{e})=\phi$ if e $\notin \mathrm{A}$.

Here $f_{A}$ is called an approximate function of the soft set $\left(f_{A}, \mathrm{E}\right)$. The set $f_{A}(\mathrm{e})$ is called e-approximate value set or e-approximate set which consists of related objects of the parameter e $\in \mathrm{E}$.

Example 1 let $U=\left\{u_{1}, u_{2}, u_{3}, u_{4}\right\}$ be a set of four shirts and $\mathrm{E}=\left\{\right.$ white $\left(e_{1}\right)$, red $\left(e_{2}\right)$,blue $\left.\left(e_{3}\right)\right\}$ be a set of parameters. If $\mathrm{A}=\left\{e_{1}, e_{2}\right\} \subseteq \mathrm{E}$. Let $f_{A}\left(e_{1}\right)=\left\{u_{1}, u_{2}\right.$, $\left.u_{3}, u_{4}\right\}$ and $f_{A}\left(e_{2}\right)=\left\{u_{1}, u_{2}, u_{3}\right\}$, then we write the soft set $\left(f_{A}, \mathrm{E}\right)=\left\{\left(e_{1},\left\{u_{1}, u_{2}, u_{3}, u_{4}\right\}\right),\left(e_{2},\left\{u_{1}\right.\right.\right.$, $\left.\left.\left.u_{2}, u_{3}\right\}\right)\right\}$ over $\mathrm{U}$ which describe the "colour of the shirts" which Mr. $\mathrm{X}$ is going to buy.

We may represent the soft set in the following form:

\begin{tabular}{|c|c|c|c|}
\hline $\mathrm{U}$ & white $\left(e_{1}\right)$ & $\operatorname{red}\left(e_{2}\right)$ & blue $\left(e_{3}\right)$ \\
\hline$u_{1}$ & 1 & 1 & 0 \\
\hline$u_{2}$ & 1 & 1 & 0 \\
\hline$u_{3}$ & 1 & 1 & 0 \\
\hline$u_{4}$ & 1 & 0 & 0 \\
\hline
\end{tabular}

\subsection{Fuzzy set [6]}

Let $\mathrm{U}$ be an initial universe, $\mathrm{E}$ be the set of all parameters 
and $\mathrm{A} \subseteq \mathrm{E}$. A pair $(\mathrm{F}, \mathrm{A})$ is called a fuzzy set over $\mathrm{U}$ where $\mathrm{F}: \mathrm{A} \rightarrow \tilde{P}(\mathrm{U})$ is a mapping from A into $\tilde{P}(\mathrm{U})$, where $\tilde{P}(\mathrm{U})$ denotes the collection of all subsets of $U$.

Example 2. Consider the example 1, here we can not express with only two real numbers 0 and 1 , we can characterized it by a membership function instead of crisp number 0 and 1 , which associate with each element a real number in the interval $[0,1]$.Then

$\left(f_{A}, \mathrm{E}\right)=\left\{f_{A}\left(e_{1}\right)=\left\{\left(u_{1}, .7\right),\left(u_{2}, .5\right),\left(u_{3}, .4\right),\left(u_{4}, .2\right)\right\}\right.$, $\left.f_{A}\left(e_{2}\right)=\left\{\left(u_{1}, .5\right),\left(u_{2}, .1\right),\left(u_{3}, .5\right)\right\}\right\}$ is the fuzzy soft set representing the "colour of the shirts" which $\mathrm{Mr}$. $\mathrm{X}$ is going to buy.

We may represent the fuzzy soft set in the following form :

\begin{tabular}{|c|c|c|c|}
\hline $\mathrm{U}$ & white $\left(e_{1}\right)$ & $\operatorname{red}\left(e_{2}\right)$ & blue $\left(e_{3}\right)$ \\
\hline$u_{1}$ & .7 & .5 & 0 \\
\hline$u_{2}$ & .5 & .1 & 0 \\
\hline$u_{3}$ & .4 & .5 & 0 \\
\hline$u_{4}$ & .2 & 0 & 0 \\
\hline
\end{tabular}

\subsection{Fuzzy Soft Matrices (FSM) [5]}

Let $\left(f_{A}, \mathrm{E}\right)$ be fuzzy soft set over $\mathrm{U}$. Then a subset of $\mathrm{U} \mathrm{x}$ $\mathrm{E}$ is uniquely defined by

$R_{A}=\left\{(\mathrm{u}, \mathrm{e}): \mathrm{e} \in \mathrm{A}, \mathrm{u} \in f_{A}(\mathrm{e})\right\}$,

which is called relation form of $\left(f_{A}, \mathrm{E}\right)$.

The characteristic function of $R_{A}$ is written by

$\mu_{R_{A}}: \mathrm{U} \mathrm{x} \mathrm{E} \rightarrow[0,1]$, where $\mu_{R_{A}}(\mathrm{u}, \mathrm{e}) \in[0,1]$ is the membership value of $\mathrm{u} \in \mathrm{U}$ for each e $\in \mathrm{U}$.

If $\mu_{i j}=\mu_{R_{A}}\left(u_{i}, e_{j}\right)$, we can define a matrix

$$
\left[\mu_{i j}\right]_{m X n}=\left[\begin{array}{cccc}
\mu_{11} & \mu_{12} & \ldots & \mu_{1 n} \\
\mu_{21} & \mu_{22} & \cdots & \mu_{2 n} \\
\vdots & \vdots & \vdots & \vdots \\
\mu_{m 1} & \mu_{m 2} & \cdots & \mu_{m n}
\end{array}\right]
$$

which is called an $\mathrm{m} \times \mathrm{n}$ soft matrix of the soft set $\left(f_{A}, \mathrm{E}\right)$ over U.

Therefore we can say that a fuzzy soft set $\left(f_{A}, \mathrm{E}\right)$ is uniquely characterized by the matrix $\left[\mu_{i j}\right]_{m X n}$ and both concepts are interchangeable.

Example 3. Assume that $U=\left\{u_{1}, u_{2}, u_{3}, u_{4}, u_{5}\right.$, $\left.u_{6}\right\}$ is a universal set and $\mathrm{E}=\left\{e_{1}, e_{2}, e_{3}, e_{4}\right\}$ is a set of all parameters. If $\mathrm{A} \subseteq \mathrm{E}=\left\{e_{1}, e_{2}, e_{3}\right\}$ and

$$
\begin{gathered}
f_{A}\left(e_{1}\right)=\left\{\left(u_{1}, .3\right),\left(u_{2}, .4\right),\left(u_{3}, .6\right),\left(u_{4}, .1\right),\left(u_{5}, .6\right),\right. \\
\left(u_{6}, 5\right\} \\
f_{A}\left(e_{2}\right)=\left\{\left(u_{1}, .2\right),\left(u_{2}, .5\right),\left(u_{3}, .7\right),\left(u_{4}, .3\right),\left(u_{5}, .7\right),\right. \\
\left.\left(u_{6}, 1\right)\right\} \\
f_{A}\left(e_{3}\right)=\left\{\left(u_{1}, .5\right),\left(u_{2}, .2\right),\left(u_{3}, .5\right),\left(u_{4}, .6\right),\left(u_{5}, .7\right),\right. \\
\left.\left(u_{6}, .3\right)\right\}
\end{gathered}
$$

Then the fuzzy soft set $\left(f_{A}, \mathrm{E}\right)$ is a parameterized family $\left\{f_{A}\left(e_{1}\right), f_{A}\left(e_{2}\right), f_{A}\left(e_{3}\right)\right\}$ of all fuzzy sets over $\mathrm{U}$.

Hence the fuzzy soft matrix $\left[\mu_{i j}\right]$ can be written as

$$
\left[\mu_{i j}\right]=\left[\begin{array}{cccc}
.3 & .2 & .5 & 0 \\
.4 & .5 & .2 & 0 \\
.6 & .7 & .5 & 0 \\
.1 & .3 & .6 & 0 \\
.6 & .7 & .7 & 0 \\
.5 & .1 & .3 & 0
\end{array}\right]
$$

\section{Intuitionistic Fuzzy Soft Matrix Theory}

\subsection{Intuitionistic Fuzzy Soft Matrix ( IFSM) [5]}

Let $\mathrm{U}$ be an initial universe, $\mathrm{E}$ be the set of parameters and A $\subseteq$ E. Let ] Let $\left(f_{A}, \mathrm{E}\right)$ be an Intuitionistic fuzzy soft set ( IFSS) over $\mathrm{U}$. Then a subset of $\mathrm{U} \times \mathrm{E}$ is uniquely defined by

$R_{A}=\left\{(\mathrm{u}, \mathrm{e}): \mathrm{e} \in \mathrm{A}, \mathrm{u} \in f_{A}(\mathrm{e})\right\}$,

which is called relation form of $\left(f_{A}, \mathrm{E}\right)$. The membership and non-membership functions of are written by

$\mu_{R_{A}}: \mathrm{U} \times \mathrm{E} \rightarrow[0,1]$ and $v_{R_{A}}: \mathrm{U} \times \mathrm{E} \rightarrow[0,1]$ where $\mu_{R_{A}}:(\mathrm{u}, \mathrm{e}) \in[0,1]$ and $v_{R_{A}}:(\mathrm{u}, \mathrm{e}) \in[0,1]$ are the membership value and non membership value of $u \in U$ for each e $\in E$.

If $\left(\mu_{i j}, v_{i j}\right)=\left(\mu_{R_{A}}\left(u_{i}, e_{j}\right), v_{R_{A}}\left(u_{i}, e_{j}\right)\right)$, we can define a matrix

$\left[\mu_{i j}, v_{i j}\right]_{m X n}=$

$\left[\begin{array}{cccc}\left(\mu_{11}, v_{11}\right) & \left(\mu_{12}, v_{12}\right) & \ldots & \left(\mu_{1 n}, v_{1 n}\right) \\ \left(\mu_{21}, v_{21}\right) & \left(\mu_{22}, v_{22}\right) & \ldots & \left(\mu_{2 n}, v_{2 n}\right) \\ \vdots & \vdots & \vdots & \vdots \\ \left(\mu_{m 1}, v_{m 1}\right) & \left.\mu_{m 2}, v_{m 2}\right) & \ldots & \left(\mu_{m n}, v_{m n}\right)\end{array}\right]$, which is

called

an $\mathrm{m} \times \mathrm{n}$ IFSM of the $\operatorname{IFSS}\left(f_{A}, \mathrm{E}\right)$ over U. Therefore, we can say that $\operatorname{IFSS}\left(f_{A}, \mathrm{E}\right)$ is uniquely characterized by the matrix $\left[\mu_{i j}, v_{i j}\right]_{m X n}$ and both concepts are interchangeable. The set of all $\mathrm{m} \times \mathrm{n}$ IFS matrices will be denoted by $\mathrm{IFSM}_{m \times n}$.

Example 4. Let $\mathrm{U}=\left\{u_{1}, u_{2}, u_{3}, u_{4}, u_{5}, u_{6}\right\}$ is a universal set and $\mathrm{E}=\left\{e_{1}, e_{2}, e_{3}, e_{4}\right\}$ is a set of parameters. If $\mathrm{A}=\left\{e_{1}, e_{2}, e_{3}\right\} \subseteq \mathrm{E}$ and

$f_{A}\left(e_{1}\right)=\left\{\left(u_{1}, .3, .4\right),\left(u_{2}, .5, .4\right),\left(u_{3}, .4, .5\right)\right.$, $\left.\left(u_{4}, .6,3\right),\left(u_{5}, 8, .1\right),\left(u_{6}, .7, .2\right)\right\}$

$f_{A}\left(e_{2}\right)=\left\{\left(u_{1}, .4, .5\right),\left(u_{2}, .6 \quad, .2\right),\left(u_{3}, 1,0\right)\right.$, $\left.\left(u_{4}, .6,2\right),\left(u_{5}, .3, .4\right),\left(u_{6}, .5, .4\right)\right\}$

$f_{A}\left(e_{3}\right)=\left\{\left(u_{1}, .6, .2\right),\left(u_{2}, 1,0\right) \quad\left(u_{3}, .9 \quad, .1\right)\right.$, $\left.\left(u_{4}, .4,2\right),\left(u_{5}, 6,6,4\right),\left(u_{6}, .7, .3\right)\right\}$

Then the IFS set $\left(f_{A}, \mathrm{E}\right)$ is a parameterized family $\left\{f_{A}\left(e_{1}\right), f_{A}\left(e_{2}\right), f_{A}\left(e_{3}\right)\right\}$ of all IFS sets over $\mathrm{U}$.

Hence IFSM $\left[\left(\mu_{i j}, v_{i j}\right)\right]$ can be written as

$$
\left[\left(\mu_{i j}, v_{i j}\right)\right]=\left[\begin{array}{cccc}
(.3, .4) & (.4, .5) & (.6, .2) & (0,0) \\
(.5, .4) & (.6, .2) & (1,0) & (0,0) \\
(.4, .5) & (1,0) & (.9, .1) & (0,0) \\
(.6, .3) & (.6, .2) & (.4, .2) & (0,0) \\
(.8, .1) & (.3, .4) & (.6, .4) & (0,0) \\
(.7, .2) & (.5, .4) & (.7, .3) & (0,0)
\end{array}\right]
$$




\subsection{Intuitionistic Fuzzy Soft Zero Matrix [5]}

Let $\tilde{A}=\left[\left(\mu_{i j}^{\tilde{A}}, v_{i j}^{\tilde{A}}\right)\right] \in \operatorname{IFSM}_{m \times n}$

Then $\tilde{A}$ is called a Zero IFSM denoted by $\tilde{0}=[(0,0)]$, if $\mu_{i j}^{\tilde{A}}=0$ and $v_{i j}^{\tilde{A}}=0$ for all $\mathrm{i}$ and $\mathrm{j}$.

\subsection{Intuitionistic Fuzzy Soft $\mu$-Universal Matrix}

An Intuitionistic fuzzy soft matrix of order $\mathrm{m} x \mathrm{n}$ is said to be an Intuitionistic Fuzzy Soft $\mu$-Universal Matrix if $\mu_{i j}^{\tilde{A}}$ $=1$ and $v_{i j}^{\tilde{A}}=0$ for all $\mathrm{i}$ and $\mathrm{j}$. It is denoted by $\tilde{I}$.

\subsection{Intuitionistic Fuzzy Soft v -Universal Matrix}

An Intuitionistic fuzzy soft matrix of order $\mathrm{m} \mathrm{x} \mathrm{n}$ is said to be an Intuitionistic Fuzzy Soft $v$-Universal Matrix if $\mu_{i j}^{\tilde{A}}$ $=0$ and $v_{i j}^{\tilde{A}}=1$ for all $\mathrm{i}$ and $\mathrm{j}$. It is denoted by $I$.

\subsection{Intuitionistic Fuzzy Soft Sub Matrix}

Let $\tilde{A}=\left[\left(\mu_{i j}^{\tilde{A}}, v_{i j}^{\tilde{A}}\right)\right], \tilde{B}=\left[\left(\mu_{i j}^{\tilde{B}}, v_{i j}^{\tilde{B}}\right)\right] \in \operatorname{IFSM}_{m X n}$. Then $\tilde{A}$ is said to be intuitionistic fuzzy soft sub matrix of $\tilde{B}$ denoted by $\tilde{A} \subseteq \tilde{B}$ if $\mu_{i j}^{\tilde{A}} \leq \mu_{i j}^{\widetilde{B}}$ and $v_{i j}^{\tilde{A}} \geq v_{i j}^{\widetilde{B}}$ for all $\mathrm{i}$ and $\mathrm{j}$.

\subsection{Intuitionistic Fuzzy Soft Super Matrix}

Let $\tilde{A}=\left[\left(\mu_{i j}^{\tilde{A}}, v_{i j}^{\tilde{A}}\right)\right], \tilde{B}=\left[\left(\mu_{i j}^{\tilde{B}}, v_{i j}^{\tilde{B}}\right)\right] \in \operatorname{IFSM}_{m X n}$. Then $\tilde{A}$ is said to be intuitionistic fuzzy soft super matrix of $\tilde{B}$ denoted by $\tilde{A} \supseteq \widetilde{B}$ if $\mu_{i j}^{\tilde{A}} \geq \mu_{i j}^{\tilde{B}}$ and $v_{i j}^{\tilde{A}} \leq v_{i j}^{\tilde{B}}$ for all $\mathrm{i}$ and $\mathrm{j}$.

\subsection{Intuitionistic Fuzzy Soft Equal Matrix}

Let $\tilde{A}=\left[\left(\mu_{i j}^{\tilde{A}}, v_{i j}^{\tilde{A}}\right)\right], \tilde{B}=\left[\left(\mu_{i j}^{\tilde{B}}, v_{i j}^{\tilde{B}}\right)\right] \in \operatorname{IFSM}_{m X n}$. Then $\tilde{A}$ is said to be equal $\tilde{B}$ denoted by $\tilde{A}=\tilde{B}$ if $\mu_{i j}^{\tilde{A}}=$ $\mu_{i j}^{\tilde{B}}$ and $v_{i j}^{\tilde{A}}=v_{i j}^{\tilde{B}}$ for all $\mathrm{i}$ and $\mathrm{j}$.

\subsection{Union of Intuitionistic Fuzzy Soft Matrices}

Let $\tilde{A}=\left[\left(\mu_{i j}^{\tilde{A}}, v_{i \tilde{A}}^{\tilde{A}}\right)\right], \tilde{B}=\left[\left(\mu_{i j}^{\tilde{B}}, v_{i \tilde{B}}^{\tilde{B}}\right)\right] \in \operatorname{IFSM}_{m X n}$. Then Union $\tilde{A}$ and $\tilde{B}$ denoted by $\tilde{A} \widetilde{\cup} \tilde{B}$ is defined as $\tilde{A}$ $\widetilde{\mathrm{U}} \tilde{B}=\left[\max \left\{\mu_{i j}^{\tilde{A}}, \mu_{i j}^{\tilde{B}}\right\}, \min \left\{v_{i j}^{\widetilde{A}}, v_{i j}^{\tilde{B}}\right\}\right]$ for all $\mathrm{i}$ and $\mathrm{j}$.

\subsection{Intersection of Intuitionistic Fuzzy Soft Matrices}

Let $\tilde{A}=\left[\left(\mu_{i j}^{\tilde{A}}, v_{i j}^{\tilde{A}}\right)\right], \tilde{B}=\left[\left(\mu_{i j}^{\tilde{B}}, v_{i j}^{\tilde{B}}\right)\right] \in \operatorname{IFSM}_{m X n}$. Then intersection $\tilde{A}$ and $\tilde{B}$ denoted by $\tilde{A} \widetilde{\cap} \tilde{B}$ is defined as $\tilde{A} \tilde{\cap} \tilde{B}=\left[\min \left\{\mu_{i j}^{\tilde{A}}, \mu_{i j}^{\tilde{B}}\right\}, \max \left\{v_{i j}^{\tilde{A}}, v_{i j}^{\tilde{B}}\right\}\right]$ for all $\mathrm{i}$ and $\mathrm{j}$.

\subsection{Complement of Intuitionistic Fuzzy Soft Matrix}

Let $\tilde{A}=\left[\left(\mu_{i j}^{\tilde{A}}, v_{i j}^{\tilde{A}}\right)\right] \in \operatorname{IFSM}_{m X n}$. Then Complement of $\tilde{A}$ denoted by $\tilde{A}^{0}$ is defined as $\tilde{A}^{0}=\left[\left(v_{i j}^{\tilde{A}}, \mu_{i j}^{\tilde{A}}\right)\right]$ for all $\mathrm{i}$ and $\mathrm{j}$.

Proposition1. Let $\tilde{A}=\left[\left(\mu_{i j}^{\tilde{A}}, v_{i j}^{A}\right)\right], \tilde{B}=\left[\left(\mu_{i j}^{\tilde{B}}, v_{i j}^{\widetilde{B}}\right)\right]$ $\in \operatorname{IFSM}_{m X n}$. Then De Morgan's type results are true which can be written as:
a) $(\tilde{A} \widetilde{\cup} \tilde{B})^{0}=\tilde{A}^{0} \widetilde{\cap} \tilde{B}^{0}$
b) $(\tilde{A} \tilde{\cap} \tilde{B})^{0}=\tilde{A}^{0} \widetilde{\cup} \tilde{B}^{0}$

Proof: a) For all $\mathrm{i}$ and $\mathrm{j}$ we have,

$(\tilde{A} \widetilde{\mathrm{U}} \tilde{B})^{0}=\left(\left[\left(\mu_{i j}^{\tilde{A}}, v_{i j}^{\tilde{A}}\right)\right] \widetilde{\mathrm{U}}\left[\left(\mu_{i j}^{\tilde{B}}, v_{i j}^{\tilde{B}}\right)\right]\right)^{0}$

$=\left[\max \left\{\mu_{i j}^{\tilde{A}}, \mu_{i j}^{\tilde{B}}\right\}, \min \left\{v_{i j}^{\tilde{A}}, v_{i j}^{\tilde{B}}\right\}\right]^{0}$

$=\left[\min \left\{v_{i j}^{\tilde{A}}, v_{i j}^{\tilde{B}}\right\}, \max \left\{\mu_{i j}^{\tilde{A}}, \mu_{i j}^{\tilde{B}}\right\}\right]$

$=\left[\left(v_{i j}^{\tilde{A}}, \mu_{i j}^{\tilde{A}}\right)\right] \widetilde{\cap}\left[\left(v_{i j}^{\tilde{B}}, \mu_{i j}^{\widetilde{B}}\right)\right]$

$=\tilde{A}^{0} \widetilde{\cap} \tilde{B}^{0} \square$

The result b) can be proved in similar way.

Proposition2. Let $\tilde{A}=\left[\left(\mu_{i j}^{\tilde{A}}, v_{i j}^{\tilde{A}}\right)\right], \tilde{B}=\left[\left(\mu_{i j}^{\tilde{B}}, v_{i j}^{\tilde{B}}\right)\right]$ $\in \operatorname{IF~SM}_{m X n}$. Then i) $\left(\tilde{A}^{0} \widetilde{\cap} \widetilde{B}^{0}\right)^{0}=\tilde{A} \widetilde{U} \tilde{B}$ ii) $\left(\tilde{A}^{0} \tilde{\mathrm{U}} \tilde{B}^{0}\right)^{0}=\tilde{A} \tilde{\cap} \tilde{B}$

iii) $\left(\tilde{A}^{0}+\tilde{B}^{0}\right)^{0}=\tilde{A}$. $\tilde{B}$ iii) $\left(\tilde{A}^{0} \cdot \tilde{B}^{0}\right)^{0}=\tilde{A}+\tilde{B}$

Proposition3. Let $\tilde{A}=\left[\left(\mu_{i j}^{\tilde{A}}, v_{i j}^{\tilde{A}}\right)\right] \in \operatorname{IFSM}_{m X n}$. Then

a) $\left(\left(\tilde{A}^{0}\right)\right)^{0}=\tilde{A}$ f) $\tilde{A} \tilde{\cap} I=\tilde{A}$

b) $(\tilde{I})^{0}=I$ g) $\tilde{A} \tilde{\cap} \tilde{A}=\tilde{A}$

c) $(I)^{0}=\tilde{I}$ h) $\tilde{A} \tilde{\cap} \tilde{I}=\tilde{A}$

d) $\tilde{A} \tilde{U} \tilde{A}=\tilde{A}$ i) $\tilde{A} \tilde{\cap} \underbrace{I}_{w}=\underbrace{}_{w}$

e) $\tilde{A} \tilde{U} \tilde{I}=\tilde{I}$

\subsection{Intuitionistic Fuzzy Soft Square Matrix}

Let $\tilde{A}=\left[\left(\mu_{i j}^{\tilde{A}}, v_{i j}^{\tilde{A}}\right)\right] \in \operatorname{IFSM}_{m X n}$. Then $\tilde{A}$ is said to be Intuitionistic Fuzzy Soft Square Matrix if $\mathrm{m}=\mathrm{n}$ for all $\mathrm{i}$ and $\mathrm{j}$.

\subsection{Intuitionistic Fuzzy Soft Row Matrix}

Let $\tilde{A}=\left[\left(\mu_{i j}^{\tilde{A}}, v_{i j}^{\tilde{A}}\right)\right] \in \operatorname{IFSM}_{m X n}$. Then $\tilde{A}$ is said to be Intuitionistic Fuzzy Soft Row Matrix if $n=1$ for all $i$ and $j$.

\subsection{Intuitionistic Fuzzy Soft Column Matrix}

Let $\tilde{A}=\left[\left(\mu_{i j}^{\tilde{A}}, v_{i j}^{\tilde{A}}\right)\right] \in \operatorname{IFSM}_{m X n}$. Then $\tilde{A}$ is said to be Intuitionistic Fuzzy Soft Column matrix if $\mathrm{m}=1$ for all $\mathrm{i}$ and $\mathrm{j}$.

\subsection{Intuitionistic Fuzzy Soft Diagonal Matrix}

Let $\tilde{A}=\left[\left(\mu_{i j}^{\tilde{A}}, v_{i j}^{\tilde{A}}\right)\right] \in \operatorname{IFSM}_{m X n}$. Then $\tilde{A}$ is said to be intuitionistic Fuzzy Soft Diagonal Matrix if $\mathrm{m}=\mathrm{n}$ and $\mathrm{i}=\mathrm{j}$.

\subsection{Max-Min Product of Intuitionistic Fuzzy Soft Matrices}

Let $\tilde{A}=\left[\left(\mu_{i j}^{\tilde{A}}, v_{i j}^{\tilde{A}}\right)\right] \in \operatorname{IFSM}_{m X n}, \tilde{B}=\left[\left(\mu_{i j}^{\tilde{B}}, v_{i j}^{\tilde{B}}\right)\right]$ $\in \mathrm{IFSM}_{n X p}$. Then the Max-Min Product of $\tilde{A}$ and $\tilde{B}$ denoted by $\tilde{A} * \tilde{B}$ is defined as $\tilde{A} * \tilde{B}=\left[\max -\min \left(\mu_{i j}^{\tilde{A}}\right.\right.$, $\left.\left.\mu_{i j}^{\widetilde{B}}\right), \min -\max \left(v_{i j}^{\tilde{A}}, v_{i j}^{\widetilde{B}}\right)\right]$ for all $\mathrm{i}$ and $\mathrm{j}$.

\subsection{Scalar Multiplication of Intuitionistic Fuzzy Soft Matrix}


Let $\tilde{A}=\left[\left(\mu_{i j}^{\tilde{A}}, v_{i j}^{\tilde{A}}\right)\right] \in \operatorname{IFSM}_{m X n}$ and k be a scalar .Then the Scalar Multiplication of Intuitionistic Fuzzy Soft Matrix $\tilde{A}$ by the scalar $\mathrm{k}$ denoted by $\mathrm{k} \tilde{A}$ defined as

$\mathrm{k} \tilde{A}=\left[\left(\mathrm{k} \mu_{i j}^{\tilde{A}}, \mathrm{k} v_{i j}^{\tilde{A}}\right)\right]$, where $0 \leq \mathrm{k} \leq 1$, for all $\mathrm{i}$ and $\mathrm{j}$.

\subsection{Operators of Intuitionistic Fuzzy Soft Matrices}

Then

Let $\tilde{A}=\left[\left(\mu_{i j}^{\tilde{A}}, v_{i j}^{\tilde{A}}\right)\right], \tilde{B}=\left[\left(\mu_{i j}^{\tilde{B}}, v_{i j}^{\tilde{B}}\right)\right] \in \operatorname{IFSM}_{m X n}$.

IFSM $\tilde{C}=\left[\left(\mu_{i j}^{\tilde{C}}, v_{i j}^{\tilde{C}}\right)\right]$ is called

a) the "."(product) operation of $\tilde{A}$ and $\tilde{B}$,denoted by $\tilde{C}=\tilde{A} . \tilde{B}$ if $\mu_{i j}^{\tilde{C}}=\mu_{i j}^{\tilde{A}} \cdot \mu_{i j}^{\tilde{B}}$ and $v_{i j}^{\tilde{C}}=v_{i j}^{\tilde{A}}+v_{i j}^{\tilde{B}}-v_{i j}^{\tilde{A}}$. $v_{i j}^{\tilde{B}}$ for all $\mathrm{i}$ and $\mathrm{j}$.

b) the "+"(Probabilistic sum) operation of $\tilde{A}$ and $\tilde{B}$, denoted by $\tilde{C}=\tilde{A}+\tilde{B}$ if $\mu_{i j}^{\tilde{C}}=\mu_{i j}^{\tilde{A}}+\mu_{i j}^{\tilde{B}}-\mu_{i j}^{\tilde{A}}$. $\mu_{i j}^{\tilde{B}}$ and $v_{i j}^{C}=v_{i j}^{\tilde{A}} \cdot v_{i j}^{\tilde{B}}$ for all $\mathrm{i}$ and $\mathrm{j}$.

c) the "@”(Arithmetic Mean) operation of $\tilde{A}$ and $\tilde{B}$,denoted by $\tilde{C}=\tilde{A} @ \tilde{B}$ if $\mu_{i j}^{\tilde{C}}=\frac{\mu_{i j}^{\widetilde{A}}+\mu_{i j}^{\widetilde{B}}}{2}$ and $v_{i j}^{\tilde{C}}=$ $\frac{v_{i j}^{\widetilde{A}}+v_{i j}^{\widetilde{B}}}{2}$ for all $\mathrm{i}$ and $\mathrm{j}$.

d) the "@w” ( Weighted Arithmetic Mean) operation of $\tilde{A}$ and $\tilde{B}$,denoted by $\tilde{C}=\tilde{A}$ @ ${ }^{w} \tilde{B}$ if $\mu_{i j}^{\tilde{C}}=$ $\frac{w_{1} \mu_{i j}^{\widetilde{A}}+w_{2} \mu_{i j}^{\widetilde{B}}}{w_{1}+w_{2}}, v_{i j}^{\tilde{C}}=\frac{w_{1} v_{i j}^{\widetilde{A}}+w_{2} v_{i j}^{\widetilde{B}}}{w_{1}+w_{2}}$, for all i and j. $w_{1}>0$, $w_{2}>0$

e) the "\$" (Geometric Mean) operation of $\tilde{A}$ and $\tilde{B}$, denoted by $\tilde{C}=\tilde{A} \$ \tilde{B}$ if $\mu_{i j}^{\tilde{C}}=\sqrt{\mu_{i j}^{\tilde{A}} \cdot \mu_{i j}^{\tilde{B}}}$ and $v_{i j}^{\tilde{C}}=$ $\sqrt{v_{i j}^{\tilde{A}} \cdot v_{i j}^{\tilde{B}}}$ for all $\mathrm{i}$ and $\mathrm{j}$.

f) the " $\$$ "” (Weighted Geometric Mean) operation of $\tilde{A}$ and $\tilde{B}$,denoted by $\tilde{C}=\tilde{A} \quad \$^{w} \quad \tilde{B}$ if $\mu_{i j}^{\tilde{C}}=$ $\left(\left(\mu_{i j}^{\tilde{A}}\right)^{w_{1}} \cdot\left(\mu_{i j}^{\tilde{B}}\right)^{w_{2}}\right)^{\frac{1}{w_{1}+w_{2}}} \quad v_{i j}^{C} \quad$ and $=$ $\left(\left(v_{i j}^{\tilde{A}}\right)^{w_{1}} \cdot\left(v_{i j}^{\tilde{E}}\right)^{w_{2}}\right)^{\frac{1}{w_{1}+w_{2}}}$ for all $\mathrm{i}$ and $\mathrm{j} \cdot w_{1}>0, w_{2}>$ 0

g) the “®”(Harmonic Mean) operation of $\tilde{A}$ and $\tilde{B}$,denoted by $\tilde{C}=\tilde{A} \bowtie \tilde{B}$ if $\mu_{i j}^{\tilde{C}}=2 \cdot \frac{\mu_{i j}^{\widetilde{A}} \cdot \mu_{i j}^{\widetilde{B}}}{\mu_{i j}^{\widetilde{A}}+\mu_{i j}^{\tilde{B}}}$ and $v_{i j}^{\tilde{C}}=$ 2. $\frac{v_{i j}^{\widetilde{A}} \cdot v_{i j}^{\widetilde{B}}}{v_{i j}^{\tilde{A}}+v_{i j}^{\widetilde{B}}}$ for all $\mathrm{i}$ and $\mathrm{j} . w_{1}>0, w_{2}>0$

h) the " $\bowtie^{w}$ " ( Weighted Harmonic Mean) operation of

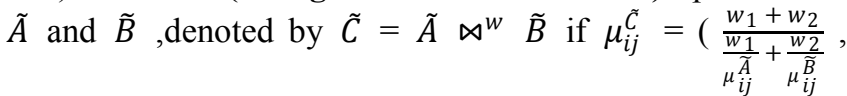
$v_{i j}^{C}=\frac{w_{1}+w_{2}}{\frac{w 1}{\tilde{A}}+\frac{w 2}{\tilde{\tilde{B}}}}$ for all $\mathrm{i}$ and $\mathrm{j}$.

Example5. Let $\tilde{A}=\left[\left(\mu_{i j}^{\tilde{A}}, v_{i j}^{\tilde{A}}\right)\right], \tilde{B}=\left[\left(\mu_{i j}^{\tilde{B}}, v_{i j}^{\tilde{B}}\right)\right] \in$ $\mathrm{IFSM}_{3 \times 2}$, where

$$
\tilde{A}=\left[\begin{array}{ll}
(.3, .2) & (.4, .5) \\
(.6, .4) & (.5, .4) \\
(.5, .3) & (.7, .2)
\end{array}\right] \text { and } \tilde{B}=\left[\begin{array}{ll}
(.3, .4) & (.6, .4) \\
(.4, .2) & (.3, .7) \\
(.7, .1) & (.6, .4)
\end{array}\right] \text {. }
$$

$$
\left(\begin{array}{lll}
\tilde{A}^{0} & \widetilde{\cup} & \tilde{B}^{0}
\end{array}\right)^{0}=\left[\begin{array}{ll}
(.3, .4) & (.4, .5) \\
(.4, .4) & (.3, .7) \\
(.5, .3) & (.6, .4)
\end{array}\right]=\tilde{A} \widetilde{\cap} \tilde{B}
$$

Proposition4. Let $\tilde{A}=\left[\left(\mu_{i j}^{\tilde{A}}, v_{i j}^{\tilde{A}}\right)\right], \widetilde{B}=\left[\left(\mu_{i j}^{\tilde{B}}, v_{i j}^{\tilde{B}}\right)\right]$ $\in \mathrm{IFSM}_{m X n}$. Then

i) $\left(\tilde{A}^{0} @^{w} \tilde{B}^{0}\right)^{0}=\tilde{A} @^{w} \tilde{B}$ ii) $\left(\tilde{A}^{0} \$^{w} \tilde{B}^{0}\right)^{0}=\tilde{A} \$^{w}$ $\tilde{B}$

iii) $\left(\tilde{A}^{0} \bowtie^{w} \tilde{B}^{0}\right)^{0}=\tilde{A} \bowtie^{w} \tilde{B}$

Proof: Let $\tilde{A}=\left[\left(\mu_{i j}^{\tilde{A}}, v_{i j}^{\tilde{A}}\right)\right], \tilde{B}=\left[\left(\mu_{i j}^{\tilde{B}}, v_{i j}^{\tilde{B}}\right)\right] \in$ $\operatorname{IFSM}_{m X n}$ and $w_{1}>0, w_{2}>0$.

$\left(\tilde{A}^{0} @^{w} \tilde{B}^{0}\right)^{0}$

$=\left(\left[\left(v_{i j}^{\tilde{A}}, \mu_{i j}^{\tilde{A}}\right)\right] @^{w}\left[\left(v_{i j}^{\tilde{B}}, \mu_{i j}^{\tilde{B}}\right)\right]\right)^{0}$

$=\left[\left(\frac{w_{1} v_{i j}^{\widetilde{A}}+w_{2} v_{i j}^{\tilde{B}}}{w_{1}+w_{2}}, \frac{w_{1} \mu_{i j}^{\widetilde{A}}+w_{2} \mu_{i j}^{\tilde{B}}}{w_{1}+w_{2}}\right)\right]^{0}$

$=\left[\left(\frac{w_{1} \mu_{i j}^{\widetilde{A}}+w_{2} \mu_{i j}^{\widetilde{B}}}{w_{1}+w_{2}}, \frac{w_{1} v_{i j}^{\widetilde{A}}+w_{2} v_{i j}^{\widetilde{B}}}{w_{1}+w_{2}}\right)\right]$

$=\tilde{A} @^{w} \tilde{B}$

Similar proof for others.

\section{Proposition5.( Commutative law)}

Let $\tilde{A}=\left[\left(\mu_{i j}^{\tilde{A}}, v_{i j}^{\tilde{A}}\right)\right], \tilde{B}=\left[\left(\mu_{i j}^{\tilde{B}}, v_{i j}^{\tilde{B}}\right)\right] \in \operatorname{IFSM}_{m X n}$. Then

i) $\tilde{A} \widetilde{U} \tilde{B}=\tilde{B}$ U $\tilde{A}$ iii) $\tilde{A}+\tilde{B}=\tilde{B}+\tilde{A}$

ii) $\tilde{A} \widetilde{\cap} \tilde{B}=\tilde{B} \widetilde{\cap} \tilde{A}$ iv) $\tilde{A} . \tilde{B}=\tilde{B} . \tilde{A}$

Proof: For all $\mathrm{i}$ and $\mathrm{j}$,

i) $\tilde{A} \widetilde{U} \tilde{B}=\left[\left(\max \left\{\mu_{i j}^{\tilde{A}}, \mu_{i j}^{\tilde{B}}\right\}, \min \left\{v_{i j}^{\tilde{A}}, v_{i j}^{\tilde{B}}\right\}\right)\right]$

$=\left[\left(\max \left\{\mu_{i j}^{\tilde{B}}, \mu_{i j}^{\tilde{A}}\right\}, \min \left\{v_{i j}^{\tilde{B}}, v_{i j}^{\tilde{A}}\right\}\right)\right]$

$=\tilde{B} \tilde{U} \tilde{A}$

Validity: Since $0 \leq \mu_{i j}^{\tilde{A}}, \mu_{i j}^{\tilde{B}} \leq 1,0 \leq v_{i j}^{\tilde{A}}, v_{i j}^{\tilde{B}} \leq 1$, $\max \left\{\mu_{i j}^{\tilde{B}}, \mu_{i j}^{\tilde{A}}\right\} \leq 1, \min \left\{v_{i j}^{\tilde{A}}, v_{i j}^{\tilde{B}}\right\} \leq 1$.

ii) $\tilde{A} \widetilde{\cap} \tilde{B}=\left[\left(\min \left\{\mu_{i j}^{\tilde{A}}, \mu_{i j}^{\tilde{B}}\right\}, \max \left\{v_{i j}^{\tilde{A}}, v_{i j}^{\tilde{B}}\right\}\right)\right]$

$=\left[\left(\min \left\{\mu_{i j}^{\tilde{B}}, \mu_{i j}^{\tilde{A}}\right\}, \max \left\{v_{i j}^{\tilde{B}}, v_{i j}^{\tilde{A}}\right\}\right)\right]$

$=\tilde{B} \widetilde{\cap} \tilde{A}$

Validity : Same as (i)

Validity: Same as viii) when $w_{1}=w_{2}$

Similarly, we can prove the other results and also the validity.

Proposition6.( Commutative laws on the base of weight)

Let $\tilde{A}=\left[\left(\mu_{i j}^{\tilde{A}}, v_{i j}^{\tilde{A}}\right)\right], \tilde{B}=\left[\left(\mu_{i j}^{\tilde{B}}, v_{i j}^{\tilde{B}}\right)\right] \in \operatorname{IFSM}_{m X n}$. Then

i) $\tilde{A} @^{w} \tilde{B}=\tilde{B} @^{w} \tilde{A}$

ii) $\tilde{A} \bowtie^{w} \tilde{B}=\tilde{B} \bowtie^{w} \tilde{A}$

iii) $\tilde{A} \$^{w} \quad \tilde{B}=\tilde{B} \$^{w} \tilde{A}$

Proof: For all $\mathrm{i}$ and $\mathrm{j}$ and $w_{1}>0, w_{2}>0$,

$\tilde{A} @^{w} \tilde{B}=\left[\left(\frac{w_{1} \mu_{i j}^{\widetilde{A}}+w_{2} \mu_{i j}^{\widetilde{B}}}{w_{1}+w_{2}}, \frac{w_{1} v_{i j}^{\widetilde{A}}+w_{2} v_{i j}^{\widetilde{B}}}{w_{1}+w_{2}}\right)\right]$

$=\left[\left(\frac{w_{2} \mu_{i j}^{\widetilde{B}}+w_{1} \mu_{i j}^{\widetilde{A}}}{w_{2}+w_{1}}, \frac{w_{2} v_{i j}^{\widetilde{B}}+w_{1} v_{i j}^{\widetilde{A}}}{w_{2}+w_{1}}\right)\right]$

$=\widetilde{B} @^{w} \tilde{A} \square$

Similar proof for others.

Proposition7.( Associative law)

Let $\tilde{A}=\left[\left(\mu_{i j}^{\tilde{A}}, v_{i j}^{\tilde{A}}\right)\right], \tilde{B}=\left[\left(\mu_{i j}^{\tilde{B}}, v_{i j}^{\tilde{B}}\right)\right], \tilde{C}=\left[\left(\mu_{i j}^{\tilde{C}}\right.\right.$, $\left.\left.v_{i j}^{\tilde{C}}\right)\right] \in \operatorname{IFSM}_{m X n}$. Then

i) $(\tilde{A} \widetilde{U} \tilde{B}) \tilde{U} \tilde{C}=\tilde{A} \widetilde{U}(\tilde{B} \widetilde{U} \tilde{C})$ 
ii) $(\tilde{A} \widetilde{\cap} \tilde{B}) \widetilde{\cap} \tilde{C}=\tilde{A} \widetilde{\cap}(\widetilde{B} \widetilde{\cap} \tilde{C})$

iii) $(\tilde{A}+\tilde{B})+\tilde{C}=\tilde{A}+(\tilde{B}+\tilde{C})$

iv) $(\tilde{A} \cdot \tilde{B}) \cdot \tilde{C}=\tilde{A} \cdot(\tilde{B} \cdot \tilde{C})$

v) $(\tilde{A} @ \tilde{B}) @ \tilde{C} \neq \tilde{A} @(\tilde{B} @ \tilde{C})$

vi) $(\tilde{A} \$ \tilde{B}) \$ \tilde{C} \neq \tilde{A} \$(\tilde{B} \$ \tilde{C})$

vii) $(\tilde{A} \bowtie \tilde{B}) \bowtie \tilde{C} \neq \tilde{A} \bowtie(\tilde{B} \bowtie \tilde{C})$

Proof : For all $\mathrm{i}$ and $\mathrm{j}$,

i) $(\tilde{A} \widetilde{U} \tilde{B}) \widetilde{U} \tilde{C}=\left[\left(\max \left\{\mu_{i j}^{\tilde{A}}, \mu_{i j}^{\tilde{B}}\right\}, \min \left\{v_{i j}^{\tilde{A}}\right.\right.\right.$, $\left.\left.\left.v_{i j}^{\tilde{B}}\right\}\right)\right] \tilde{U}\left[\left(\mu_{i j}^{\tilde{C}}, v_{i j}^{\tilde{C}}\right)\right]$

$=\left[\left(\max \left\{\left(\mu_{i j}^{\tilde{A}}, \mu_{i j}^{\tilde{B}}\right), \mu_{i j}^{C}\right\}, \min \left\{\left(v_{i j}^{\tilde{A}}, v_{i j}^{\tilde{B}}\right)\right.\right.\right.$, $\left.\left.\left.v_{i j}^{\tilde{C}}\right\}\right)\right]$

$=\left[\left(\max \left\{\mu_{i j}^{\tilde{A}},\left(\mu_{i j}^{\tilde{B}}, \mu_{i j}^{\tilde{C}}\right)\right\}, \min \left\{v_{i j}^{\tilde{A}},\left(v_{i j}^{\tilde{B}}\right.\right.\right.\right.$, $\left.\left.\left.\left.v_{i j}^{\tilde{C}}\right)\right\}\right)\right]$

$=\tilde{A} \widetilde{U}(\tilde{B} \widetilde{\cup} \tilde{C})$

Validity : Since $0 \leq \mu_{i j}^{\tilde{A}}, \mu_{i j}^{\tilde{B}}, \mu_{i j}^{\tilde{C}} \leq 1,0 \leq v_{i j}^{\tilde{A}}, v_{i j}^{\tilde{B}}$, $v_{i j}^{\tilde{C}} \leq 1$, 1

$\max \left\{\mu_{i j}^{\tilde{A}}, \mu_{i j}^{\tilde{B}}, \mu_{i j}^{\tilde{C}}\right\} \leq 1, \min \left\{v_{i j}^{\tilde{A}}, v_{i j}^{\tilde{B}}, \mu_{i j}^{\tilde{C}}\right\} \leq$

ii) $(\tilde{A} \widetilde{\cap} \tilde{B}) \widetilde{\cap} \tilde{C}=\left[\left(\min \left\{\mu_{i j}^{\tilde{A}}, \mu_{i j}^{\tilde{B}}\right\}, \max \left\{v_{i j}^{\tilde{A}}\right.\right.\right.$, $\left.\left.\left.v_{i j}^{\tilde{B}}\right\}\right)\right] \tilde{\cap}\left[\left(\mu_{i j}^{C}, v_{i j}^{C}\right)\right]$

$=\left[\left(\min \left\{\left(\mu_{i j}^{\tilde{A}}, \mu_{i j}^{\tilde{B}}\right), \mu_{i j}^{C}\right\}, \max \left\{\left(v_{i j}^{\tilde{A}}, v_{i j}^{\tilde{B}}\right)\right.\right.\right.$, $\left.\left.\left.v_{i j}^{C}\right\}\right)\right]$

$=\left[\left(\min \left\{\mu_{i j}^{\tilde{A}},\left(\mu_{i j}^{\tilde{B}}, \mu_{i j}^{\tilde{C}}\right)\right\}, \max \left\{v_{i j}^{\tilde{A}},\left(v_{i j}^{\tilde{B}}\right.\right.\right.\right.$, $\left.\left.\left.\left.v_{i j}^{\tilde{C}}\right)\right\}\right)\right]$

$=\tilde{A} \widetilde{\cap}(\tilde{B} \tilde{\cap} \tilde{C})$

Validity : Same as (i)

Similarly, we can verify the other results.

Proposition8. (Distributive law) Let $\tilde{A}=\left[\left(\mu_{i j}^{\tilde{A}}, v_{i j}^{\tilde{A}}\right)\right]$, $\tilde{B}=\left[\left(\mu_{i j}^{\tilde{B}}, v_{i j}^{\tilde{B}}\right)\right]$ and $\tilde{C}=\left[\left(\mu_{i j}^{\tilde{C}}, v_{i j}^{\tilde{C}}\right)\right] \in \operatorname{IFSM}_{m X n}$. Then

i) $\tilde{A} \widetilde{\cap}(\tilde{B} \widetilde{U} \tilde{C})=(\tilde{A} \widetilde{\cap} \tilde{B}) \widetilde{U}(\tilde{A} \widetilde{\cap} \tilde{C})$

ii) $(\tilde{A} \widetilde{\cap} \tilde{B}) \widetilde{U} \tilde{C}=(\tilde{A} \widetilde{U} \tilde{C}) \widetilde{\cap}(\tilde{B} \widetilde{U} \tilde{C})$

iii) $\tilde{A} \widetilde{\mathrm{U}}(\tilde{B} \widetilde{\cap} \tilde{C})=(\tilde{A} \widetilde{\mathrm{U}} \tilde{B}) \widetilde{\cap}(\tilde{A} \widetilde{\mathrm{U}} \tilde{C})$

iv) $(\tilde{A} \tilde{\cup} \tilde{B}) \tilde{\cap} \tilde{C}=(\tilde{A} \widetilde{\cap} \tilde{C}) \widetilde{U}(\tilde{B} \widetilde{\cap} \tilde{C})$

v) $(\tilde{A} \widetilde{\cap} \tilde{B}) @ \tilde{C}=(\tilde{A} @ \tilde{C}) \widetilde{n}(\tilde{B} @ \tilde{C})$

vi) $(\tilde{A} \widetilde{\cap} \tilde{B}) \bowtie \tilde{C}=(\tilde{A} \bowtie \tilde{C}) \tilde{\cap}(\tilde{B} \bowtie \tilde{C})$

vii) $(\tilde{A} \widetilde{U} \tilde{B})+\tilde{C}=(\tilde{A}+\tilde{C}) \widetilde{U}(\tilde{B}+\tilde{C})$

viii) ( $\tilde{A} \widetilde{\cup} \tilde{B}) \cdot \tilde{C}=(\tilde{A} \cdot \tilde{C}) \widetilde{U}(\tilde{B} \cdot \tilde{C})$

ix) $\tilde{A} \widetilde{U}(\tilde{B} @ \tilde{C})=(\tilde{A} \widetilde{\mathrm{U}}) @(\tilde{A} \widetilde{\mathrm{U}} \tilde{C})$

$\mathrm{x})(\tilde{A} \widetilde{\mathrm{U}} \tilde{B}) \bowtie \tilde{C}=(\tilde{A} \bowtie \tilde{C}) \tilde{U}(\tilde{B} \bowtie \tilde{C})$

xi) $\tilde{A} @(\tilde{B} \widetilde{U} \tilde{C})=(\tilde{A} @ \tilde{B}) \widetilde{U}(\tilde{A} @ \tilde{C})$

xii) $\tilde{A} @(\tilde{B} \widetilde{\cap} \tilde{C})=(\tilde{A} @ \tilde{B}) \tilde{\cap}(\tilde{B} @ \tilde{C})$

xiii) $\tilde{A} \$(\tilde{B} \widetilde{U} \tilde{C})=(\tilde{A} \$ \tilde{B}) \tilde{U}(\tilde{A} \$ \tilde{C})$

$\operatorname{xiv})(\tilde{A} \tilde{\mathrm{U}} \tilde{B}) \$ \tilde{C}=(\tilde{A} \$ \tilde{C}) \tilde{\mathrm{U}}(\tilde{B} \$ \tilde{C})$

xv) $\tilde{A} \cdot(\tilde{B} @ \tilde{C})=(\tilde{A} . \tilde{B}) @(\tilde{A} \cdot \tilde{C})$

xvi) $\tilde{A} \widetilde{U}(\tilde{B} \bowtie \tilde{C})=(\tilde{A} \widetilde{\mathrm{U}} \tilde{B}) \bowtie(\tilde{A} \widetilde{\mathrm{U}} \tilde{C})$

xvii) $\tilde{A} \bowtie(\tilde{B} \widetilde{U} \tilde{C})=(\tilde{A} \bowtie \tilde{B}) \widetilde{U}(\tilde{A} \bowtie \tilde{C})$

xviii) $\tilde{A} \$(\tilde{B} \tilde{\cap} \tilde{C})=(\tilde{A} \$ \tilde{B}) \tilde{\cap}(\tilde{A} \$ \tilde{C})$

$\operatorname{xix})(\tilde{A} \widetilde{\cap} \tilde{B}) \$ \tilde{C}=(\tilde{A} \$ \tilde{C}) \tilde{\cap}(\tilde{B} \$ \tilde{C})$
Proof : Let $\tilde{A}=\left[\left(\mu_{i j}^{\tilde{A}}, v_{i j}^{\tilde{A}}\right)\right], \tilde{B}=\left[\left(\mu_{i j}^{\tilde{B}}, v_{i j}^{\tilde{B}}\right)\right]$ and $\tilde{C}$ $=\left[\left(\mu_{i j}^{C}, v_{i j}^{C}\right)\right] \in \operatorname{IFSM}_{m X n}$.

Then

i) $\tilde{A} \widetilde{\cap}(\tilde{B} \widetilde{\cup} \tilde{C})$

$=\left[\left(\mu_{i j}^{\tilde{A}}, v_{i j}^{\tilde{A}}\right)\right] \widetilde{\cap}\left[\left(\max \left\{\mu_{i j}^{\tilde{B}}, \mu_{i j}^{\tilde{C}}\right\}, \min \left\{v_{i j}^{\tilde{B}}, v_{i j}^{\tilde{C}}\right\}\right)\right]$

$=\left[\left(\min \left(\mu_{i j}^{\tilde{A}}, \max \left\{\mu_{i j}^{\tilde{B}}, \mu_{i j}^{\tilde{C}}\right\}\right), \max \left(v_{i j}^{\tilde{A}}, \min \left\{v_{i j}^{\tilde{B}}, v_{i j}^{\tilde{C}}\right\}\right)\right)\right]$ $(\tilde{A} \widetilde{\cap} \tilde{B}) \widetilde{U}(\tilde{A} \widetilde{\cap} \tilde{C})$

$=\left[\left(\min \left\{\mu_{i j}^{\tilde{A}}, \mu_{i j}^{\tilde{B}}\right\}, \max \left\{v_{i j}^{\tilde{A}}, v_{i j}^{\tilde{B}}\right\}\right)\right] \widetilde{U}$ $\left[\left(\min \left\{\mu_{i j}^{\tilde{A}}, \mu_{i j}^{\tilde{C}}\right\}, \max \left\{v_{i j}^{\tilde{A}}, v_{i j}^{\tilde{C}}\right\}\right)\right]$

$=\left[\left(\max \left(\min \left\{\mu_{i j}^{\tilde{A}}, \mu_{i j}^{\tilde{B}}\right\}, \min \left\{\mu_{i j}^{\tilde{A}}, \mu_{i j}^{\tilde{C}}\right\}\right), \min (\max \right.\right.$ $\left.\left.\left.\left\{v_{i j}^{\tilde{A}}, v_{i j}^{\tilde{B}}\right\}, \max \left\{v_{i j}^{\tilde{A}}, v_{i j}^{\tilde{C}}\right\}\right)\right)\right]$

$=\left[\left(\max \left(\mu_{i j}^{\tilde{A}}, \min \left\{\mu_{i j}^{\tilde{B}}, \mu_{i j}^{\tilde{C}}\right\}\right), \min \left(v_{i j}^{\tilde{A}}, \max \left\{v_{i j}^{\tilde{A}}\right.\right.\right.\right.$, $\left.\left.\left.\left.v_{i j}^{\tilde{C}}\right\}\right)\right)\right]$

$=\left[\left(\min \left(\mu_{i j}^{\tilde{A}}, \max \left\{\mu_{i j}^{\tilde{B}}, \mu_{i j}^{\tilde{C}}\right\}\right), \max \left(v_{i j}^{\tilde{A}}, \min \left\{v_{i j}^{\tilde{B}}, v_{i j}^{\tilde{C}}\right\}\right)\right)\right]$ Hence $\tilde{A} \widetilde{\cap}(\tilde{B} \widetilde{\mathrm{U}} \tilde{C})=(\tilde{A} \widetilde{\cap} \tilde{B}) \widetilde{U}(\tilde{A} \tilde{\cap} \tilde{C}) \square$

iii ) $\tilde{A} \widetilde{U}(\tilde{B} \widetilde{\cap} \tilde{C})$

$=\left[\left(\mu_{i j}^{\tilde{A}}, v_{i j}^{\tilde{A}}\right)\right] \widetilde{U}\left[\left(\min \left\{\mu_{i j}^{\tilde{B}}, \mu_{i j}^{\tilde{C}}\right\}, \max \left\{v_{i j}^{\tilde{B}}, v_{i j}^{\tilde{C}}\right\}\right)\right]$

$=\left[\left(\max \left(\mu_{i j}^{\tilde{A}}, \min \left\{\mu_{i j}^{\tilde{B}}, \mu_{i j}^{\tilde{C}}\right\}\right), \min \left(v_{i j}^{\tilde{A}}, \max \left\{v_{i j}^{\tilde{B}}, v_{i j}^{\tilde{C}}\right\}\right)\right)\right]$ $(\tilde{A} \widetilde{\cup} \tilde{B}) \widetilde{\cap}(\tilde{A} \widetilde{\cup} \tilde{C})$

$=\left[\left(\max \left\{\mu_{i j}^{\tilde{A}}, \mu_{i j}^{\tilde{B}}\right\}, \min \left\{v_{i j}^{\tilde{A}}, v_{i j}^{\tilde{B}}\right\}\right] \widetilde{\cap}\left[\left(\max \left\{\mu_{i j}^{\tilde{A}}\right.\right.\right.\right.$, $\left.\left.\left.\mu_{i j}^{\tilde{C}}\right\}, \min \left\{v_{i j}^{\tilde{A}}, v_{i j}^{\tilde{C}}\right\}\right)\right]$

$=\left[\left(\min \left(\max \left\{\mu_{i j}^{\tilde{A}}, \mu_{i j}^{\tilde{B}}\right\}, \max \left\{\mu_{i j}^{\tilde{A}}, \mu_{i j}^{C}\right\}\right), \max (\min \right.\right.$ $\left.\left.\left.\left\{v_{i j}^{\tilde{A}}, v_{i j}^{\tilde{B}}\right\}, \min \left\{v_{i j}^{\tilde{A}}, v_{i j}^{C}\right\}\right)\right)\right]$

$=\left[\left(\min \left(\mu_{i j}^{\tilde{A}}, \max \left\{\mu_{i j}^{\tilde{B}}, \mu_{i j}^{\tilde{C}}\right\}\right), \max \left(v_{i j}^{\tilde{A}}, \min \left\{v_{i j}^{\tilde{A}}\right.\right.\right.\right.$, $\left.\left.\left.\left.v_{i j}^{\tilde{C}}\right\}\right)\right)\right]$

$=\left[\left(\max \left(\mu_{i j}^{\tilde{A}}, \min \left\{\mu_{i j}^{\tilde{B}}, \mu_{i j}^{\tilde{C}}\right\}\right), \min \left(v_{i j}^{\tilde{A}}, \max \left\{v_{i j}^{\tilde{B}}, v_{i j}^{\tilde{C}}\right\}\right)\right)\right]$ Hence, $\tilde{A} \widetilde{\mathrm{U}}(\tilde{B} \widetilde{\cap} \tilde{C})=(\tilde{A} \widetilde{\mathrm{U}} \tilde{B}) \widetilde{\cap}(\tilde{A} \widetilde{\mathrm{U}} \tilde{C})$. $\square$ Similarly, we can prove other results.

Example6. Let $\tilde{A}=\left[\left(\mu_{i j}^{\tilde{A}}, v_{i j}^{\tilde{A}}\right)\right], \tilde{B}=\left[\left(\mu_{i j}^{\tilde{B}}, v_{i j}^{\tilde{B}}\right)\right], \tilde{C}$ $=\left[\left(\mu_{i j}^{C}, v_{i j}^{C}\right)\right] \in \mathrm{IFSM}_{2 \times 2}$, where

$\tilde{A}=\left[\begin{array}{ll}(.3, .2) & (.4, .5) \\ (.5, .3) & (.7, .2)\end{array}\right], \tilde{B}=\left[\begin{array}{ll}(.4, .3) & (.6, .2) \\ (.7, .2) & (.3, .4)\end{array}\right], \tilde{C}=$ $\left[\begin{array}{ll}(.6, .3) & (.5, .3) \\ (.6, .4) & (.7, .2)\end{array}\right]$

i) $\tilde{A} \widetilde{\cap}\left(\begin{array}{lll}\tilde{B} & \widetilde{C} & \tilde{C}\end{array}\right)=\left[\begin{array}{ll}(.3, .3) & (.4, .5) \\ (.5, .3) & (.7, .2)\end{array}\right]=\left(\begin{array}{lll}\tilde{A} & \widetilde{\cap} & \widetilde{B}\end{array}\right) \widetilde{\mathrm{U}}$ $(\tilde{A} \widetilde{\cap} \tilde{C})$

Proposition9.(Laws of Idempotence on the base of weight)

i) $\tilde{A} @^{w} \tilde{A}=\tilde{A}$

ii) $\tilde{A} \bowtie^{w} \tilde{A}=\tilde{A}$

iii) $\tilde{A} \$^{w} \tilde{A}=\tilde{A}$

Proof: Let $\tilde{A}=\left[\left(\mu_{i j}^{\tilde{A}}, v_{i j}^{\tilde{A}}\right)\right] \in \operatorname{IFSM}_{m X n}$.

Then for all $\mathrm{i}$ and $\mathrm{j}$ and $\mathrm{w}=w_{1}=w_{2}>0$,

ii) $\tilde{A} \bowtie^{w} \tilde{A}$

$=\left[\left(\frac{w+w}{\frac{w}{\mu_{i j}^{\widetilde{A}}}+\frac{w}{\mu_{i j}^{\widetilde{A}}}},\left(\frac{w+w}{\frac{w}{\widetilde{\widetilde{A}}}+\frac{w}{v_{i j}^{\widetilde{R}}}}\right)\right]\right.$

$=\left[\left(\mu_{i j}^{\tilde{A}}, v_{i j}^{\tilde{A}}\right)\right]$

$=\tilde{A} \square$

Similar proof for others. 


\section{Application of Weighted Arithmetic Mean $\left(A_{W A M}\right)$ of Intuitionistic Fuzzy Soft Matrix in Decision Making}

In this section, we define arithmetic mean and weighted arithmetic mean of intuitionistic fuzzy soft matrix.

\subsection{Weighted Arithmetic Mean of Intuitionistic Fuzzy Soft Matrix $\left(A_{W A M}\right)$}

Let $\tilde{A}=\left[\left(\mu_{i j}^{\tilde{A}}, v_{i j}^{\tilde{A}}\right)\right] \in \operatorname{IFSM}_{m X n}$. Then Weighted Arithmetic Mean of Intuitionistic Fuzzy Soft Matrix $\tilde{A}$ denoted by $\tilde{A}_{W A M}$ is defined as $\tilde{A}_{W A M}=\left[\left(\frac{\sum_{j=1}^{n} w_{j} \mu_{i j}^{\widetilde{A}}}{\sum_{j=1}^{n} w_{j}}\right.\right.$, $\left.\left.\frac{\sum_{j=1}^{n} w_{j} v_{i j}^{\widetilde{A}}}{\sum_{j=1}^{n} w_{j}}\right)\right]$,

$w_{j}$ for $\mathrm{j}=1,2, \ldots, \mathrm{n}$ are respective weights for membership and non-membership value .

Note: - Arithmetic Mean (A.M.) of Intuitionistic Fuzzy Soft Matrix : Let $\tilde{A}=\left[\left(\mu_{i j}^{\tilde{A}}, v_{i j}^{\tilde{A}}\right)\right] \in \operatorname{IFSM}_{m X n}$. Then Arithmetic Mean of Intuitionistic Fuzzy Soft Matrix $\tilde{A}$ of membership and non-membership value denoted by $\tilde{A}_{A M}$ is defined as $\tilde{A}_{A M}=\left[\left(\frac{\sum_{j=1}^{n} \mu_{i j}^{\widetilde{A}}}{n}, \frac{\sum_{j=1}^{n} v_{i j}^{\widetilde{A}}}{n}\right)\right]$ when weights are equal .

Input : Intuitionistic fuzzy soft sets with m objects, each of which has $n$ parameters.

Output : An optimum result.

Algorithm:

Step- 1: Choose the set of parameters.

Step -2: Construct the intuitionistic fuzzy soft matrix for the set of parameters.

Step- 3: Compute the weighted arithmetic mean of membership and non-membership value of intuitionistic fuzzy soft matrix as $A_{W A M}$.

Step-4: Choose the object with highest membership value. In case of tie i.e. when more than one object with same highest membership value, choose the object with highest membership value as well as lowest non- membership value.

Example 7. Suppose a company A produces five different types of cars $c_{1}, c_{2}, c_{3}, c_{4}, c_{5}$ such that $\mathrm{U}=\left\{c_{1}\right.$, $\left.c_{2}, c_{3}, c_{4}, c_{5}\right\}$ and $\mathrm{E}=\left\{e_{1}\right.$ (comfort),$e_{2}(\operatorname{good}$ mileage $), e_{3}$ (good power steering $\left.)\right\}$ be a set of parameters . Suppose Mr. X is going to buy a car and an intuitionistic fuzzy soft matrix is constructed on the basis of the parameters as follows :

$$
\begin{aligned}
\mathrm{A} & =\left[\begin{array}{lll}
(.5, .4) & (.6, .2) & (.5, .2) \\
(.9, .1) & (.5, .1) & (.4, .3) \\
(.6, .2) & (.5, .4) & (.7, .2) \\
(.6, .2) & (.8, .2) & (.6, .4) \\
(.4, .3) & (.6, .2) & (.5, .1)
\end{array}\right] \\
\mathrm{A}_{A M} & =\left[\begin{array}{c}
(.533, .267) \\
(.6, .167) \\
(.6, .267) \\
(.667, .267) \\
(.5, .2)
\end{array}\right] \ldots \ldots \ldots \ldots .
\end{aligned}
$$

If we prefer to "comfort" of the cars and weights $.8, .1, .1$ are given on the parameters "comfort", "good mileage", "good power steering" respectively, then

$$
\mathrm{A}_{W A M}=\left[\begin{array}{c}
(.51, .36) \\
(.81, .12) \\
(.6, .22) \\
(.62, .22) \\
(.43, .27)
\end{array}\right]
$$

From the above results (1) and (2), it is clear that if we give equal preference, we have .667 is the highest membership value of (1). So $c_{4}$ car is most suitable for $\mathrm{Mr}$. $\mathrm{X}$. But if we give more preference on comfort than other parameters(good mileage, good power steering), then $c_{2}$ car is the most suitable for Mr. X .

\section{Conclusion}

In this paper, we have introduced some new operators on the base of weights (weighted A.M, weighted G.M , weighted H.M ) and properties on intuitionistic fuzzy soft matrix. Some of the associative laws and distributive properties have been proved and verified with examples. Some of the commutative laws and idempotent laws on the base of weight have been proved. Lastly we have given one elementary application for decision making problem on the basis weighted arithmetic mean. This method can be applied on other decision making problem with uncertain parameters.

\section{REFERENCES}

[1] D. Molodtsov, Soft set theory - first result, Computers and Mathematics with Applications 37(1999), 19-31.

[2] P. K. Maji , R. Biswas and A. R. Roy, Soft Set Theory, Computer and Mathematics with Applications 45(2003), 555-562.

[3] P. K. Maji , R. Biswas and A. R. Roy, An application of soft sets in a decision making problems, Computer and Mathematics with Applications 44(2002), 1077-1083.

[4] Naim Cagman and Serdar Enginoglu, Soft matrix theory and its decision making, Computers and Mathematics with Applications 59(2010), 3308-3314.

[5] B. Chetia and P.K. Das, Some results of intuitionistic fuzzy soft matrix theory, Advances in Applied Science Research (2012),3(1), 412-423.

[6] P. K. Maji, R. Biswas and A. R. Roy, "Fuzzy Soft Sets", Journal of Fuzzy Mathematics, Vol 9 , no.3 , ( 2001), pp.589 -602 .

[7] N. Cagman and S. Enginoglu, Fuzzy soft matrix theory and its application in decision making, International Journal of Fuzzy Systems, vol.9 , (2012) pp.109-119.

[8] Manas Jyoti Borah, Tridiv Jyoti Neog, Dusmanta Kumar Sut, 
Fuzzy soft matrix theory and its decision making, IJMER, vol.2 ,issue2 March-Apr, (2012) pp.121-127.

[9] Irfan Deli, Naim Cagman, Intuitionistic fuzzy parameterized soft set theory and its decision making_1301.0454v1[ math.LO] 3 Jan 2013.

[10] P.Rajarajeswari, P.Dhanalakshmi, Intuitionistic fuzzy soft matrix theory and its application in decision making, International Journal of Engineering Research \& Technology, vol. 2 , issue 4, April-2013.

[11] Babitha K.V. and Sunil Jacob John , Generalized Intuitionistic fuzzy soft sets and its applications, Gen.Math.Notes,Vol.7,No.2.December2011,pp. $1-14$ 